\title{
Balloon angioplasty or stent implantation for pulmonary vein stenosis caused by fibrosing mediastinitis: a systematic review
}

\author{
Yichao Duan, ${ }^{1,2 \#}$, Xing Zhou ${ }^{3 \#}$, Hongling Su${ }^{2}$, Kaiyu Jiang ${ }^{2}$, Wenyu Wu ${ }^{1,2}$, Xin Pan ${ }^{4}$, Guanming Qi ${ }^{5}$, \\ Yan Zhang ${ }^{6}$, Yunshan Cao ${ }^{2}$
}

${ }^{1}$ Clinical Medicine School, Ningxia Medical University, Yinchuan 750004, China; ${ }^{2}$ Department of Cardiology, ${ }^{3}$ Department of Radiology, Gansu Provincial Hospital, Lanzhou University, Lanzhou 730000, China; ${ }^{4}$ Department of Cardiology, Shanghai Chest Hospital, Shanghai Jiao Tong University, Shanghai 200000, China; ${ }^{5}$ Department of Pulmonary, Tufts Medical Center, Boston, Massachusetts, USA; ${ }^{6}$ Tianjin Key Laboratory of Retinal Functions and Diseases, Tianjin Medical University Eye Hospital, School of Optometry and Ophthalmology \& Eye Institute, Tianjin 300384, China

Contributions: (I) Conception and design: Y Cao, Y Zhang; (II) Administrative support: All authors; (III) Collection and assembly of data: K Jiang, W Wu, H Su; (IV) Data analysis and interpretation: X Zhou, Y Duan; (V) Manuscript writing: All authors; (VI) Final approval of manuscript: All authors.

"These authors contributed equally to this work.

Correspondence to: Yunshan Cao. Department of Cardiology, Gansu Provincial Hospital, Lanzhou University, Lanzhou 730000, China. Email: yunshancao@126.com; Yan Zhang. Tianjin Key Laboratory of Retinal Functions and Diseases, Tianjin Medical University Eye Hospital, School of Optometry and Ophthalmology \& Eye Institute, Tianjin 300384, China. Email: yanzhang04@tmu.edu.cn.

\begin{abstract}
Fibrosing mediastinitis (FM) is a very rare disease, often caused by histoplasmosis capsulatum, tuberculosis, sarcoidosis, autoimmunity and other diseases, such as IgG 4-related diseases. Fibrous structures in the mediastinum compress the pulmonary artery, pulmonary vein, superior vena cava, esophagus, trachea and cardiac vessels, leading to clinical symptoms. Drug therapeutic modality for pulmonary vein stenosis (PVS) caused by FM is palliative in essence and with limited efficacy, whereas surgical treatment causes high mortality. In recent years, catheter-based treatment to FM-caused PVS has emerged as a promising therapeutic modality, however, the safety and effectiveness of this modality remain unclear. Therefore, a systematic review on the safety and efficacy of the catheter-based treatment for PVS caused by FM was performed, in the hope to shed lights on the alternative therapeutic strategy to this fatal disease.
\end{abstract}

Keywords: Fibrosing mediastinitis (FM); pulmonary vein stenosis (PVS); pulmonary vein stent; catheter; balloon angioplasty

Submitted Jul 31, 2019. Accepted for publication Sep 04, 2019.

doi: $10.21037 /$ cdt.2019.09.14

View this article at: http://dx.doi.org/10.21037/cdt.2019.09.14

\section{Introduction}

Fibrosing mediastinitis (FM) is a very rare disease. It is often caused by histoplasmosis capsulatum, tuberculosis, sarcoidosis, autoimmunity and other diseases, such as IgG 4-related diseases (1-4), which lead to the celluloselike inflammatory exudation in the mediastinum and the compression of the mediastinal pulmonary artery (PA), pulmonary vein (PV), superior vena cava (SVC), esophagus, trachea and cardiac vessels (1,5-9). Compression of mediastinal structures caused by FM has been associated with high mortality (10-13) due to pulmonary hypertension and right heart failure (14).

It is reported that the average diagnostic age of FM ranges between 20 and 40 years old $(1,13,15,16)$. Histoplasma capsulatum infection is the main cause of FM in North America, but patients of histoplasma capsulatum infection rarely develop FM $(1,12)$. During an outbreak of this fungal infection in Indianapolis, only 3 of 100,000 patients with histoplasmosis developed FM (17). However, 
Dines et al. found that 11 of 31 patients $(35.5 \%)$ with mediastinal granuloma eventually developed FM (18). In Asia, particularly in developing countries, tuberculosisrelated FM may be more frequent (19-21).

The symptoms of FM reflect the mechanical compression of the involved mediastinal structures (16). For example, esophageal compression may cause dysphagia and odynophagia; compression of thoracic duct leads to chylothorax; compressed damage of recurrent laryngeal nerves and phrenic nerve generates the symptoms of hoarseness or shortness of breath. More seriously, pulmonary vascular compression can cause pulmonary hypertension, hemoptysis, refractory pleural effusion, and pulmonary edema. Moreover, SVC compression results in facial swelling, superficial venous distention, headache, hoarseness, and shortness of breath. Bronchial compression can elicit cough, dyspnea, hemoptysis, obstructive pneumonia, and even shunting due to atelectasis $(1,11,16)$.

Drug therapy for pulmonary vein stenosis (PVS) caused by FM is palliative and with limited efficacy, surgical treatment modality is accompanied with high mortality. In recent years, balloon angioplasty or stent implantation has been reported for the treatment of FM-induced PVS, but the sample size is small and the efficacy is not definitive. Therefore, a systematic review has been performed on the safety and efficacy of the catheter-based treatment for PVS caused by FM.

\section{Methods}

\section{Literature search}

A systematic search from 1st January 1947 to 10th March 2019, from PubMed, Cochrane Library, and Embase was carried out using the medical subject headings (MeSH) terms for fibrosing mediastinitis, mediastinal fibrosis, sclerosing mediastinitis, idiopathic mediastinal fibrosis with boolean terms such as OR. Search strategies were modified for each database to achieve the broadest coverage. Details of the search strategy are available in the Supplementary file 1.

\section{Selection criteria}

Patients who had been diagnosed as FM by biopsy, computed tomography scanning (CT), and magnetic resonance imaging (MRI), and had received PV stent implantation or balloon angioplasty were include in the analysis.

\section{Data extraction}

Two reviewers independently searched the databases using the identical key words. All data were extracted from the text, tables, and figures, including supplemental material. Disagreements were resolved by consensus or consulting with a third reviewer.

\section{Statistical analysis}

Continuous variables are expressed as mean \pm SD or percentage. Quantitative analysis of the results was not done due to significant heterogeneity in the design, patient population, outcome definitions among the studies as well as data missing, therefore, a narrative summary of the available evidence was presented.

\section{Results}

The process of literature search was summarized in Figure 1. A total of 584 articles on FM were retrieved. Eight studies, including two retrospective studies $(15,22)$ and six case reports (7,23-27), were analyzed. Only the information on the PV was extracted from these articles. The main characteristics of these patients were described in Table 1.

\section{Diagnosis}

There is no unified standard for the diagnosis of FM. Biopsy is the gold standard for FM diagnosis (2). It is undeniable that due to the invasiveness of biopsy, the diagnostic value of imaging examinations, such as CT and MRI (19,28-31), is increasing. The imaging features of FM include infiltrating mediastinal soft tissue (with or without calcification), compression or obstruction of mediastinal internal structures, including PA, PV, SVC, esophagus, trachea, and cardiac vessels (Figure 2) (31). Ventilation/ perfusion (V/Q) scintigraphy often indicates the perfusion defects caused by pulmonary embolism, however, this can also occur in $\mathrm{FM}(32,33)$.

Of the 31 patients receiving catheter-based treatment for FM-induced PVS, 4 cases were diagnosed by mediastinal biopsy, 1 by MRI and 25 by computed tomographic pulmonary angiography (1 was not mentioned in the 


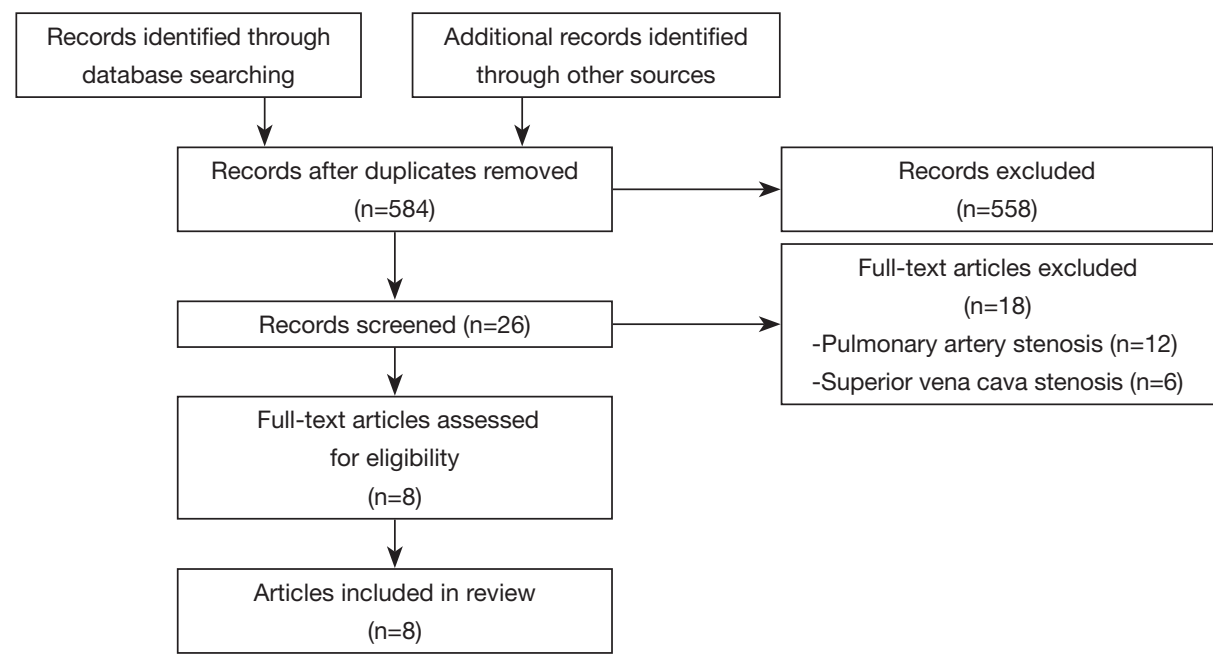

Figure 1 The process of literature selection.

Table 1 The characteristics of patients with pulmonary vein stenosis caused by fibrosing mediastinitis

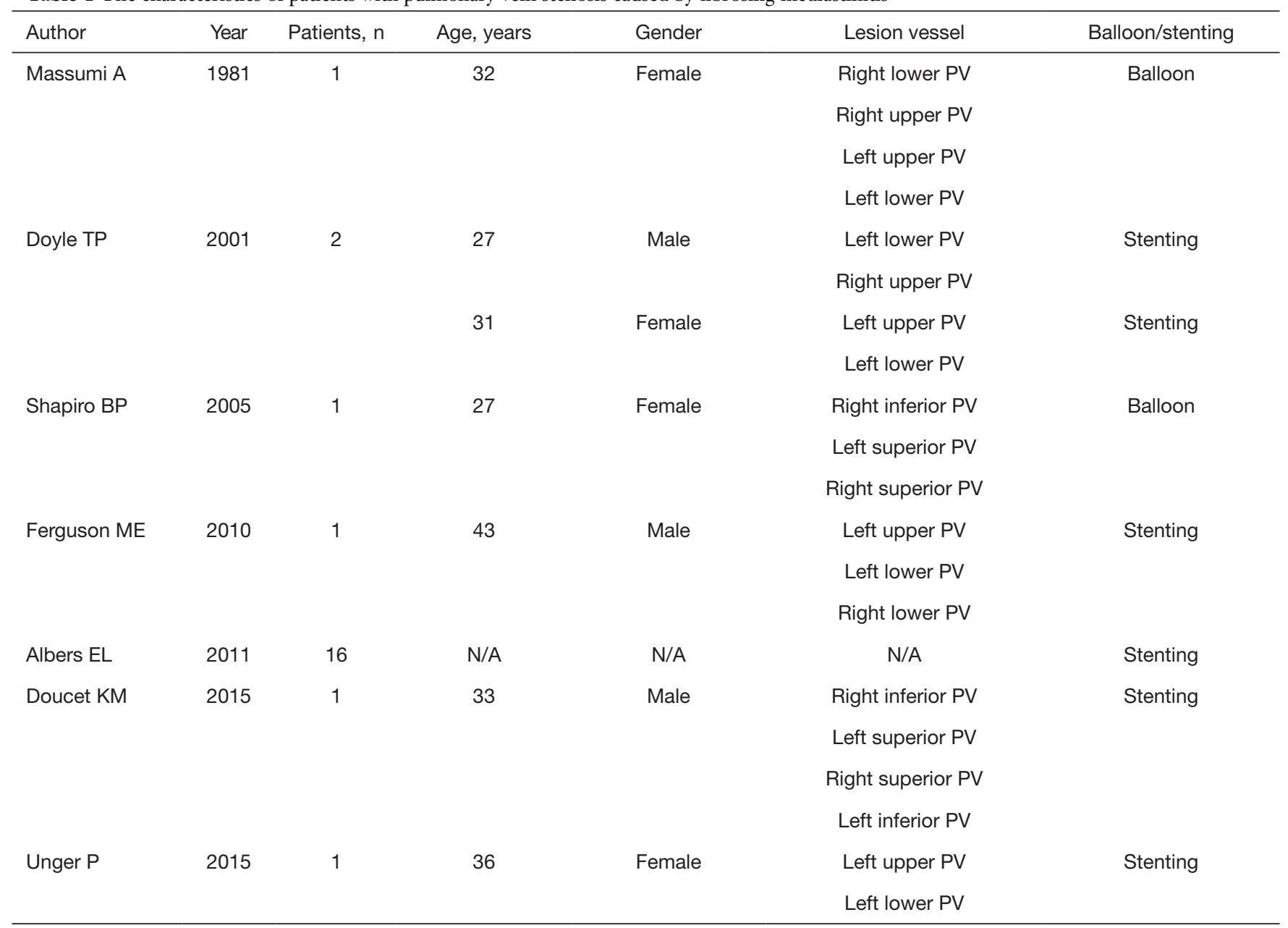

Table 1 (continued) 
Table 1 (continued)

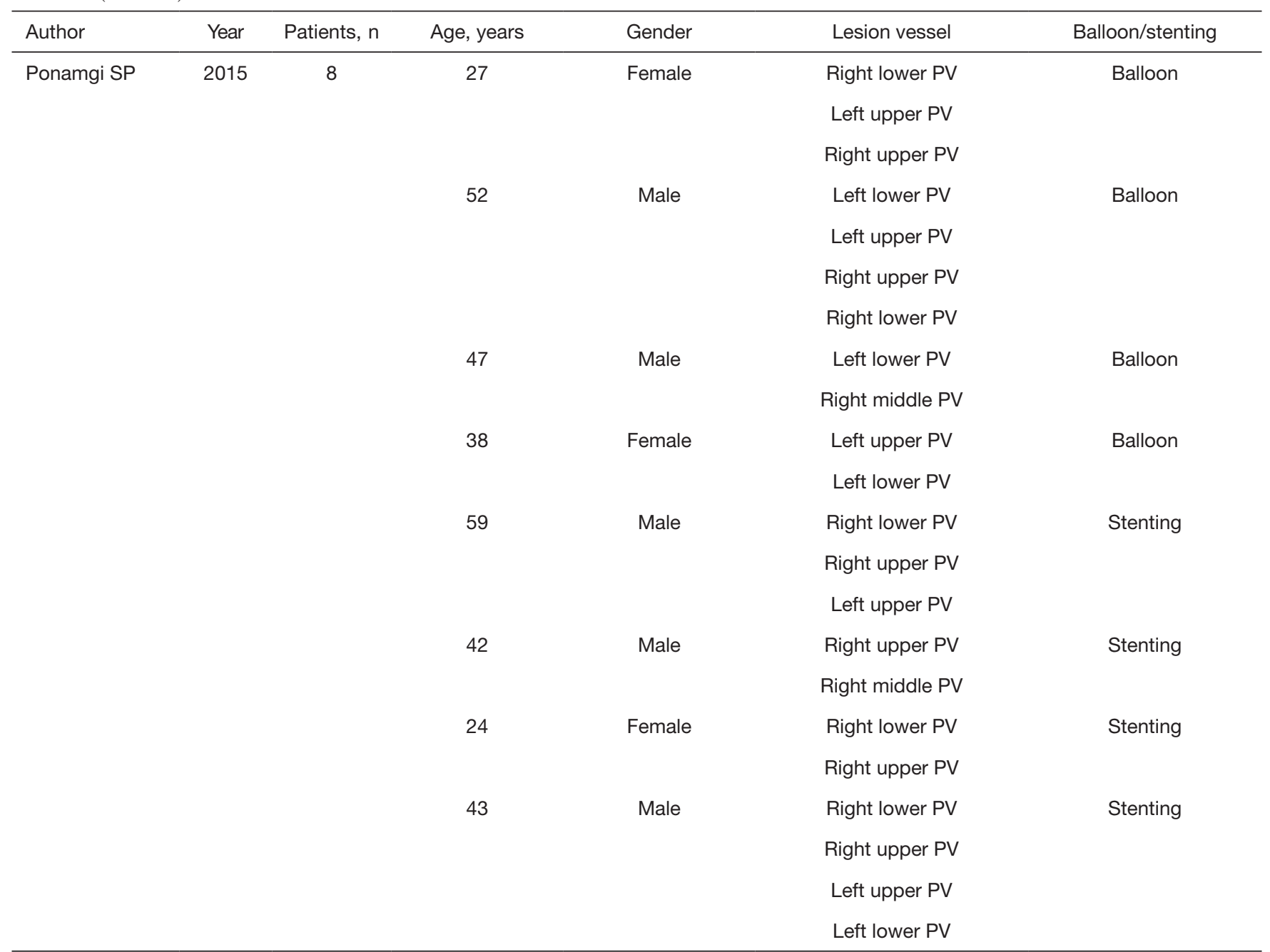

PV, pulmonary vein; N/A, not available.

article).

\section{Balloon angioplasty or stent implantation}

Catheter-based treatment included balloon angioplasty and stent implantation. Following balloon angioplasty, stenosis may recur. On the other hand, $\mathrm{PV}$ restenosis is a major complication after stent implantation. When restenosis occurs, balloon dilation or stent implantation will be performed based on the imaging examination results.

A total of 31 patients with FM-caused PVS received catheter-based treatment, of which 25 patients were subjected to stent implantation and 6 patients to balloon angioplasty.

\section{Antiplatelet or anticoagulant strategy following catheter- based treatments}

After catheter-based treatments of PVS, the antiplatelets or anticoagulants should be administered to the patients. Sixteen patients after stent implantation received anticoagulation treatment of aspirin or coumadin for a minimum of 6 months. One patient received the regimen of life-long aspirin $80 \mathrm{mg}$ and 1-month clopidogrel $75 \mathrm{mg}$. Another patient received warfarin and clopidogrel for 6 months. Other studies did not describe the detailed antiplatelet or anticoagulant strategy following PV interventions. 


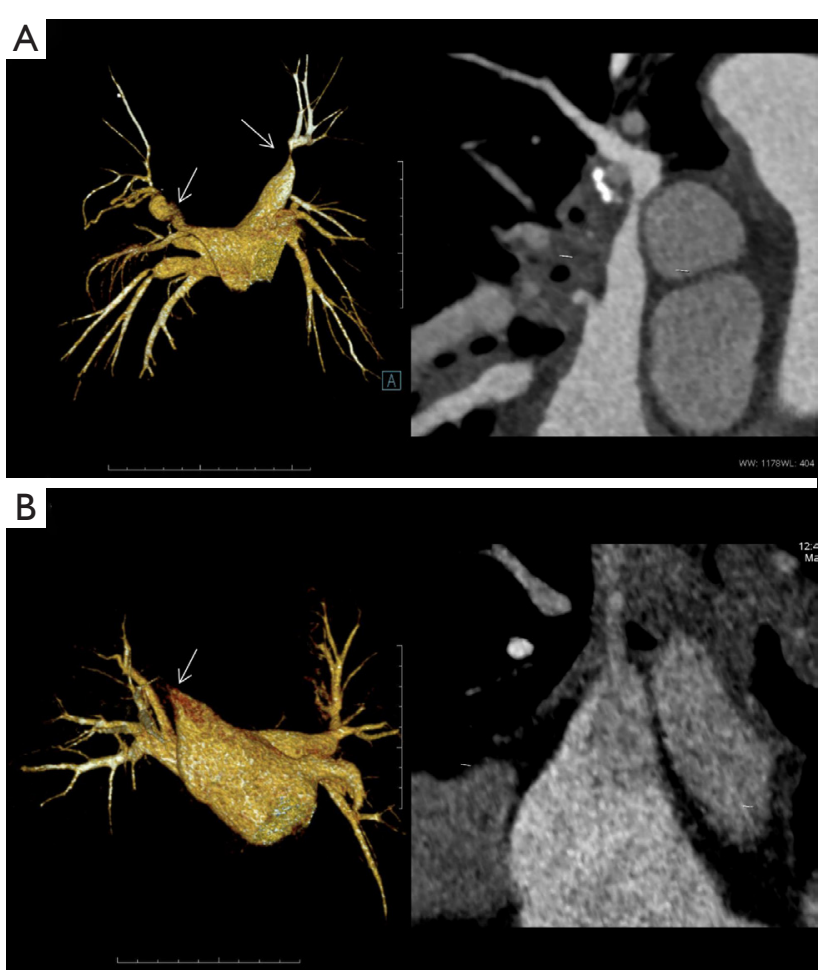

Figure 2 Representative CT venography of fibrosing mediastinitis. (A) Pulmonary vein stenosis induced by fibrosing mediastinitis; (B) pulmonary vein occlusion caused by fibrosing mediastinitis. CT, computed tomography.

\section{Clinical improvement}

Except for those who died, the patients exhibited significant improvement during the follow-up period after catheterbased treatment. The clinical improvement was mainly reflected in WHO functional classification, 6-minute walking distance, and clinical symptoms. The detailed data are shown in Table 2.

\section{Mortality}

In the study with the largest sample size (15), the patients had stenosis in PAs, PVs, and SVC, the mortality of the patients received $\mathrm{PV}$ interventions was unknown. Of the 31 patients receiving catheter-based treatments for PVS (7,15,22-27), 15 had detailed information (7,22-27). Among these 15 patients, 7 died (22-24,27).

One patient was suggestive of infection because of uncontrollable high fever. Three days following the catheter-based treatment, the clinical symptoms deteriorated rapidly and the patient succumbed to death (23). Another two patients deceased at 4 days post catheter treatments because of massive hemoptysis incapacitating ventilation function $(22,24)$. Another patient died of the deterioration of clinical symptoms without mentioning cause of death at 2 days post treatment (24). One patient died of newly diagnosed metastatic cancer at 1 month after catheter-based treatment (24); another died of a large embolic stroke with temporal lobe herniation (24); and one died of heart failure at 9 months following the catheter treatment during the operation of PV dilatation (27).

\section{Complication}

Short-term complications included bleeding from the puncture site, mild hemoptysis, phrenic nerve injury, and temporary ST segment elevation. These complications generally did not require additional treatments. Severe vascular injuries, such as PV perforation, pericardial tamponade, $\mathrm{PV}$ rupture, acute stent thrombosis complicated by stroke, intracerebral bleeding, hemothorax, stent dislodged, entailed urgent treatments and could cause periprocedural death. The main long-term complication is intra-stent restenosis.

Haemoptysis was noted in $5(16.1 \%)$ of the 31 patients who had received catheter-based treatments for PVS. Restenosis occurred in 12 (38.7\%) patients. One patient developed myocardial infarction during the operation and embolic stroke at 1 day post-operation.

\section{Discussion}

$\mathrm{FM}$ is a rare disease and $\mathrm{FM}$-induced $\mathrm{PA} / \mathrm{PV}$ stenosis can lead to pulmonary hypertension and right heart failure. Due to the limited efficacy of medicinal treatment and high risk of surgical intervention, catheter-based treatment has emerged as a promising modality to treat this increasingly fatal disease (16). Although balloon dilatation was initially performed in 1981 by Massumi et al. for PVS caused by FM (27), the efficacy and safety of the catheter-based treatment are still not clear. In this systematic review, the efficacy and safety of percutaneous PV balloon dilatation or stent for FM-caused PVS were collected from the literature and evaluated.

According to the literature, a total of 31 patients with PVS caused by FM who had undergone catheter-based treatments were included. Most of them underwent stent implantation, which was consistent with the interventional strategy of PVS induced by radiofrequency ablation for 


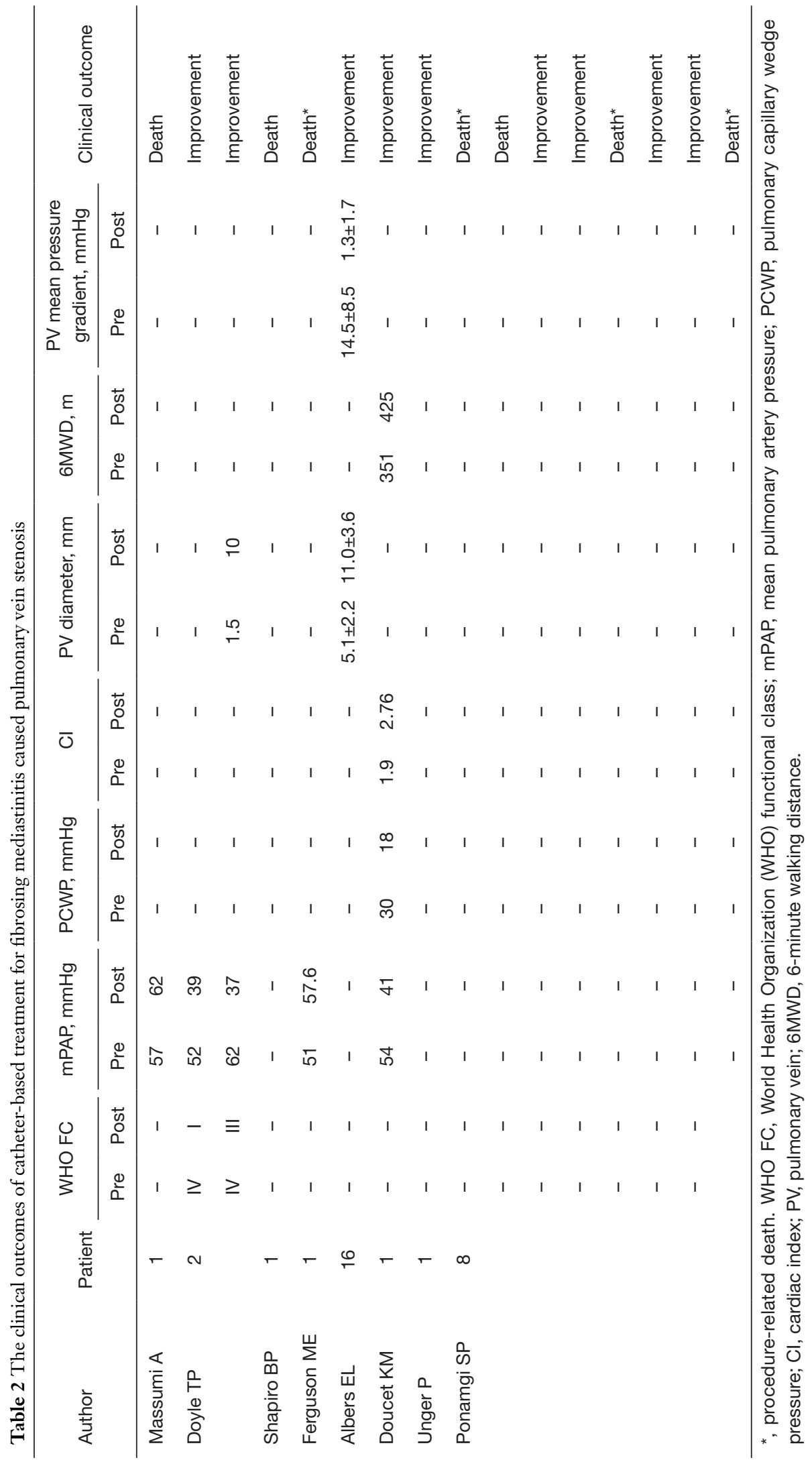


atrial fibrillation (34). Catheter-based treatments improved the hemodynamics and symptoms of the patients; however, the long-term data were absent.

In complications related to catheter-based treatments of PV caused by FM, restenosis appears to be the major one, followed by hemoptysis. For catheter-based treatment of PVS induced by radiofrequency ablation for atrial fibrillation, the restenosis rate following balloon angioplasty was $53 \%$ to $72 \%$ and that after stent implantation was $19 \%$ to $33 \%$ (34-40). The restenosis rate of stent implantation was significantly lower than that of balloon angioplasty, which supports stent implantation as the first choice for PVS caused by radiofrequency ablation for atrial fibrillation. This should also apply to PVS caused by FM. In catheter-based treatments of PVS caused by FM, some studies have shown that restenosis rates range from $43.75 \%$ to $50 \%(15,24)$. The discrepancy in the restenosis rates between the treatments for radio frequency-induced PVS and those for FMinduced PVS may be ascribed to the sample size and the patient conditions. Hemoptysis was also common after the catheter-based treatments of FM-induced PVS, nonetheless, most hemoptysis was mild.

Seven patients died after catheter-based treatment of PVS caused by FM, and four cases belonged to procedurerelated death $(22,24)$. By contrast, no procedure-related death has been reported in the catheter-based treatments of the PVS induced by radiofrequency ablation for atrial fibrillation (34-40). Therefore, the relatively high mortality of catheter-based treatments for the FM-induced PVS might be due to the limited experience during the early stage of developing the catheter-based treatments in the new indication and the small sample size available in the literature. Therefore, large-scale, multi-center, randomized controlled studies are needed to further evaluate the longterm efficacy, safety, and complications of the catheterbased treatments to the FM-induced PVS.

\section{Conclusions}

Catheter-based treatment significantly improves hemodynamic parameters and relieves symptoms of the patients with PVS caused by FM, although the long-term efficacy, safety, and restenosis rate need to be further evaluated.

\section{Acknowledgments}

None.

\section{Footnote}

Conflicts of Interest: The authors have no conflicts of interest to declare.

Ethical Statement: The authors are accountable for all aspects of the work in ensuring that questions related to the accuracy or integrity of any part of the work are appropriately investigated and resolved.

\section{References}

1. Loyd JE, Tillman BF, Atkinson JB, et al. Mediastinal fibrosis complicating histoplasmosis. Medicine (Baltimore) 1988;67:295-310.

2. Takanashi S, Akiyama M, Suzuki K, et al. IgG4-related fibrosing mediastinitis diagnosed with computed tomography-guided percutaneous needle biopsy: Two case reports and a review of the literature. Medicine (Baltimore) 2018;97:e10935.

3. Toonkel RL, Borczuk AC, Pearson GD, et al. Sarcoidosisassociated fibrosing mediastinitis with resultant pulmonary hypertension: a case report and review of the literature. Respiration 2010;79:341-5.

4. Wu Z, Jarvis H, Howard LS, et al. Post-tuberculous fibrosing mediastinitis: a review of the literature. BMJ Open Respir Res 2017;4:e000174.

5. Cardenal F, Pallares C, Vera L, et al. Superior vena cava syndrome due to tuberculous mediastinal fibrosis. Report of two cases (author's transl). Med Clin (Barc) 1979;73:103-8.

6. Chang SH, Shih CW, Lei MH. Idiopathic mediastinal fibrosis with involvement of the pulmonary vessels and left main coronary artery. Catheter Cardiovasc Interv 2012;79:1019-22.

7. Doyle TP, Loyd JE, Robbins IM. Percutaneous pulmonary artery and vein stenting: a novel treatment for mediastinal fibrosis. Am J Respir Crit Care Med 2001;164:657-60.

8. Leong DP, Dundon BK, Steele PM. Unilateral pulmonary vein stenosis secondary to idiopathic fibrosing mediastinitis. Heart 2008;94:776.

9. Lloyd TV, Johnson JC. Pulmonary artery occlusion following fibrosing mediastinitis due to histoplasmosis. Clin Nucl Med 1979;4:35-6.

10. Dunn EJ, Ulicny KS Jr, Wright CB, et al. Surgical implications of sclerosing mediastinitis. A report of six cases and review of the literature. Chest 1990;97:338-46.

11. Mathisen DJ, Grillo HC. Clinical manifestation of 
mediastinal fibrosis and histoplasmosis. Ann Thorac Surg 1992;54:1053-7; discussion 1057-8.

12. Sherrick AD, Brown LR, Harms GF, et al. The radiographic findings of fibrosing mediastinitis. Chest 1994;106:484-9.

13. Peikert T, Colby TV, Midthun DE, et al. Fibrosing mediastinitis: clinical presentation, therapeutic outcomes, and adaptive immune response. Medicine (Baltimore) 2011;90:412-23.

14. Simonneau G, Montani D, Celermajer DS, et al. Haemodynamic definitions and updated clinical classification of pulmonary hypertension. Eur Respir J 2019. doi: 10.1183/13993003.01913-2018.

15. Albers EL, Pugh ME, Hill KD, et al. Percutaneous vascular stent implantation as treatment for central vascular obstruction due to fibrosing mediastinitis. Circulation 2011;123:1391-9.

16. Fender EA, Widmer RJ, Knavel Koepsel EM, et al. Catheter based treatments for fibrosing mediastinitis. Catheter Cardiovasc Interv 2019. [Epub ahead of print].

17. Wheat LJ, Slama TG, Eitzen HE, et al. A large urban outbreak of histoplasmosis: clinical features. Ann Intern Med 1981;94:331-7.

18. Dines DE, Payne WS, Bernatz PE, et al. Mediastinal granuloma and fibrosing mediastinitis. Chest 1979;75:320-4.

19. Atasoy C, Fitoz S, Erguvan B, et al. Tuberculous fibrosing mediastinitis: CT and MRI findings. J Thorac Imaging 2001;16:191-3.

20. Ojeifo O, Gilotra NA, Kemp CD, et al. Mediastinal Fibrosis of the Pulmonary Artery Secondary to Tuberculosis. Ann Thorac Surg 2015;100:e49-50.

21. Tan R, Martires J, Kamangar N. Tuberculosis-associated Fibrosing Mediastinitis: Case Report and Literature Review. J Clin Imaging Sci 2016;6:32.

22. Ferguson ME, Cabalka AK, Cetta F, et al. Results of intravascular stent placement for fibrosing mediastinitis. Congenit Heart Dis 2010;5:124-33.

23. Shapiro BP, Sprung J, Scott K, et al. Cardiovascular collapse induced by position-dependent pulmonary vein occlusion in a patient with fibrosing mediastinitis. Anesthesiology 2005;103:661-3.

24. Ponamgi SP, DeSimone CV, Lenz CJ, et al. Catheterbased intervention for pulmonary vein stenosis due to fibrosing mediastinitis: The Mayo Clinic experience. Int J Cardiol Heart Vasc 2015;8:103-7.

25. Doucet KM, Labinaz M, Chandy G, et al. Pulmonary hypertension due to fibrosing mediastinitis treated successfully with stenting of pulmonary vein stenoses. Can
J Cardiol 2015;31:548.e5-7.

26. Unger P, Stoupel E, Briki R, et al. Successful stenting of left pulmonary veins stenosis resulting from fibrosing mediastinitis. Eur Heart J 2015;36:2623.

27. Massumi A, Woods L, Mullins CE, et al. Pulmonary venous dilatation in pulmonary veno-occlusive disease. Am J Cardiol 1981;48:585-9.

28. McNeeley MF, Chung JH, Bhalla S, et al. Imaging of granulomatous fibrosing mediastinitis. AJR Am J Roentgenol 2012;199:319-27.

29. Rodríguez E, Soler R, Pombo F, et al. Fibrosing mediastinitis: CT and MR findings. Clin Radiol 1998;53:907-10.

30. Rholl KS, Levitt RG, Glazer HS. Magnetic resonance imaging of fibrosing mediastinitis. AJR Am J Roentgenol 1985;145:255-9.

31. Garrana SH, Buckley JR, Rosado-de-Christenson ML, et al. Multimodality Imaging of Focal and Diffuse Fibrosing Mediastinitis. Radiographics 2019;39:651-67.

32. Bowman AW, Albers BK, Jain MK. Acquired Whole-lung Mismatched Perfusion Defects on Pulmonary Ventilation/ Perfusion Scintigraphy. Indian J Nucl Med 2018;33:312-6.

33. Rudkovskaia AA, Bandyopadhyay D. Intraluminal Arterial Filling Defects Misdiagnosed as Pulmonary Emboli: What Else Could They Be? Clin Chest Med 2018;39:505-13.

34. Schoene K, Arya A, Jahnke C, et al. Acquired Pulmonary Vein Stenosis After Radiofrequency Ablation for Atrial Fibrillation: Single-Center Experience in Catheter Interventional Treatment. JACC Cardiovasc Interv 2018;11:1626-32.

35. Qureshi AM, Prieto LR, Latson LA, et al. Transcatheter angioplasty for acquired pulmonary vein stenosis after radiofrequency ablation. Circulation 2003;108:1336-42.

36. Robbins IM, Colvin EV, Doyle TP, et al. Pulmonary vein stenosis after catheter ablation of atrial fibrillation. Circulation 1998;98:1769-75.

37. Prieto LR, Schoenhagen P, Arruda MJ, et al. Comparison of stent versus balloon angioplasty for pulmonary vein stenosis complicating pulmonary vein isolation. J Cardiovasc Electrophysiol 2008;19:673-8.

38. Fender EA, Widmer RJ, Hodge DO, et al. Severe Pulmonary Vein Stenosis Resulting From Ablation for Atrial Fibrillation: Presentation, Management, and Clinical Outcomes. Circulation 2016;134:1812-21.

39. Neumann T, Kuniss M, Conradi G, et al. Pulmonary vein stenting for the treatment of acquired severe pulmonary vein stenosis after pulmonary vein isolation: clinical implications after long-term follow-up of 4 years. J 
Cardiovasc Electrophysiol 2009;20:251-7.

40. Fink T, Schluter M, Heeger CH, et al. Pulmonary vein stenosis or occlusion after catheter ablation of atrial

Cite this article as: Duan $\mathrm{Y}$, Zhou $\mathrm{X}$, Su H, Jiang K, Wu W, Pan X, Qi G, Zhang Y, Cao Y. Balloon angioplasty or stent implantation for pulmonary vein stenosis caused by fibrosing mediastinitis: a systematic review. Cardiovasc Diagn Ther 2019;9(5):520-528. doi: 10.21037/cdt.2019.09.14 fibrillation: long-term comparison of drug-eluting versus large bare metal stents. Europace 2018;20:e148-55. 
Supplementary file 1: Details of the search strategy

(((Fibrosing Mediastinitis[MeSH Terms]) OR Mediastinal Fibrosis[MeSH Terms]) OR Sclerosing mediastinitis[Text Word]) OR Idiopathic mediastinal fibrosis[Text Word] 